BULl. AUSTRAL. MATH. SOC.

VOL. 34 (1986) 233-251

\title{
A WIDE PERRON INTEGRAL
}

\author{
D.N. SARKHEL
}

\begin{abstract}
In terms of an arbitrary limit process $T$, defined abstractly for real functions, we define in a novel way a $T$-continuous integral of Perron type, admitting mean value theorems, integration by parts and the analogue of the Marcinkiewicz theorem for the ordinary Perron integral. The integral is shown to include, as particular cases, the various known continuous, approximately continuous, cesàro-continuous, mean-continuous and proximally cesaro-continuous integrals of Perron and Denjoy types. An interesting generalisation of the classical Lebesgue decomposition theorem is also obtained.
\end{abstract}

\section{Introduction}

Bullen and Lee [4] gave a method of unifying various integrals of Perron type, by introducing the abstract notion of a 'derivate system'. In an endeavour to find a simpler but more revealing and more concrete unifying method leading to desirable generalisations, recently in [23] we introduced the $T$-continuous Perron integral, (TP), defined in terms of the notion of proximal variation and in terms of the limit process $T$ induced by an orderly connected topology on the real line. This marks a major modification of the perron method inasmuch as the difference of a major (upper) function and a minor (lower) function need no longer be nondecreasing. However, the (TP)-integral was shown to admit of mean value theorems, an integration by parts formula and the analogue of the important Marcinkiewicz theorem for the ordinary Perron integral ([16], (3.13), p. 253). Besides, it was shown to include, as particular cases, Received 19 November 1985.

Copyright Clearance Centre, Inc. Serial-fee code: 0004-9727/86 $\$ \mathrm{~A} 2.00+0.00$. 
various known continuous and approximately continuous integrals ([6]), [12], [13], [27]). Further, $T$ could also be taken to be the proximal limit process introduced in [22], which is more general than the approximate limit process.

But, the $(T P)$-integral could not unify the mean type continuous integrals, such as the Cesàro-Perron integrals, $C_{n} P$, of Burkill $[7,8]$, the $C_{n} D$ and $V_{n} D$ integrals of sargent $[17,18]$, the mean-continuous integrals, $G M_{n}$, of Ellis [10], and the recently introduced proximally Cesàro-continuous integral, $(P C D)$, of Nath and Bose [14]. The failure is due to the fact that mean type continuity is essentially nontopological in nature [21].

The purpose of this paper is to show (section 4) that, with new methods of proofs, the entire theory of the (TP)-integral remains valid in fact for any limit process $T$, defined abstractly (section 3 ) by assuming only the bare necessities, Quite pleasingly, with appropriate choice of $T$, the (TP)-integral now includes (section 5) also each of the mean type continuous integrals mentioned above. Besides, the (TP)-integral leads us to an interesting generalisation (Corollary 4.9.1) of the classic result that the derivative of a function of bounded variation, $V B$, is always Lebesgue integrable, which in turn leads to an extensive generalisation (Corollary 4.9.2) of the classical Lebesgue decomposition theorem ([16], p. 120). Convergence theorems for the (TP)-integral can be deduced, as usual, from those for the Lebesgue integral, and will be omitted. The (TP)-integral also admits of a simple Denjoy type constructive definition, which will be given elsewhere.

We remark in passing that, no single integral can exist which would include all the integrals mentioned above, because the two integrals $[6,7]$ of Burkill are not compatible, as shown by Ellis [11].

\section{Preliminaries}

Throughout this paper, $\mathrm{R}$ will denote the real line, $|E|$ the outer Lebesgue measure of a subset $E \subseteq \mathrm{R}$, and $E^{0}$ its interior. By $f \supseteq E \rightarrow \mathrm{R}[f \sim E \rightarrow \mathrm{R}]$ we shall mean that $f$ is an extended real valued function defined and finite at least for all [almost all] points of the 
set $E$, and then $f_{E}$ will denote the function defined on $R$ by $f_{E}(x)=f(x)$ if $x \in$ (dom $\left.f\right) \cap E$ and $f_{E}(x)=0$ otherwise. Also, we denote by ess-sup $\operatorname{su}_{E} f$ [ess-inf $E_{E} f$ ] the infimum [supremum] of the numbers $r$ such that

$$
|\{x \in E \mid f(x)>r\}|=0 \quad[|\{x \in E \mid f(x)<r\}|=0] .
$$

The function $f$ is said to satisfy the condition $(N)$ of Lusin (16], p. 224) on $E$, if $|f(H)|=0$ for every $H \subseteq E$ with $|H|=0$. For the notions of approximate continuity and derivative and of functions $V B$ and $A C$ we refer to [16].

A finite family (possibly void) of pairwise disjoint open intervals with end points on a set $E \subseteq R$ is called a subdivision of $E$. A sequence $\left\{E_{n}\right\}$. of sets whose union is $E$ is called an $E$-form with parts $E_{n}$; if, moreover, each part $E_{n}$ is closed in $E$, then the $E$-form is said to be closed. An expanding $E$-form is called an E-chain ([23], Definition 2.1).

Given $f \supseteq E \rightarrow R$ and $r>0$, we denote by $V(f, E ; r)$ the supremum of the sums $\left\{\left|f\left(b_{i}\right)-f\left(a_{i}\right)\right|\right.$ for all subdivisions $\left\{\left(a_{i}, b_{i}\right)\right\}$ of $E$ with $\sum\left(b_{i}-a_{i}\right)<r$. We define $V(f, E)=\sup _{r>0} V(f, E ; r)$ and $V(f, E ; 0)=\inf _{r>0} V(f, E ; r)$.

We note that $f$ is $V B[A C]$ on $E$ if and only if $V(f, E)<\infty$ $[V(f, E ; 0)=0]$. If $f$ is $V B[A C]$ on each part of a closed $E$-form, then $f$ is called $(V B G)[(A C G)]$ on $E$. The infimum of $\sup _{n} V\left(f, E_{n} ; 0\right)$ for all $E$-chains $\left\{E_{n}\right\}$, denoted $P V(f, E)$, is called the proximal variation of $f$ on $E$. If $P V(f, E)<\infty[P V(f, E)=0]$ then $f$ is called (PVB) [(PAC)] on $E$. For details about these notions we refer to [23], section 3 , but summarize below some of the results obtained therein for ready reference.

(2.1). For all $p, q \in \mathrm{R}$ and all $f, g \supseteq E \rightarrow \mathrm{R}$ we have $P V(p f+q g, E) \leqslant|p| \cdot P V(f, E)+|q| \cdot P V(g, E)$.

(2.2). If $P V(g, E)=0$, then $P V(f+g, E)=P V(f, E)$. 
(2.3). If $f$ and $g$ are both $(P V B)[(P A C)]$ on $E$, then $p f+q g$ is also $(P V B)[(P A C)]$ on $E$ for all $p, q \in \mathrm{R}$.

(2.4). If $|E|=0$, then $|f(\vec{E})| \leqslant P V(f, E)$. In particular, if $f$ is $(P A C)$ on $E$, then $f$ satisfies Lusin's condition $(N)$ on $E$.

(2.5). If $f$ is $(P V B)$ on $E$, then $f$ is $(V B G)$ on $E$; if, further, the set $E$ is measurable, then $f \mid E$ is measurable and $f$ has a finite approximate derivative, $(o p) f^{\prime}(x)$, at almost all points $x$ of $E$.

(2.6). If $f$ is (PAC) on each part of a closed $E$-form, then $f$ is necessarily $(P A C)$ on $E$.

(2.7). If $f$ and $g$ are both $(P A C)$ on $E$, so is $f g$.

(2.8). If $f$ is $(P V B)$ on $E$, then $P V(f, E \cap I)$ is an additive continuous function of intervals $I \subseteq \mathrm{R}$.

(2.9). If $f$ is $(A C G)$ on $E$, then $f$ is necessarily (PAC) on $E$; the converse is true if $E$ is closed and $f \mid E$ is continuous.

(2.10). Let $\left\{p_{n}\right\}$ and $\left\{q_{n}\right\}$ denote two sequences in $\mathrm{R}$ such that $\sum\left|p_{n}\right|<\infty$ and $q_{i} \neq q_{j}$ for $i \neq j$, and let $F: \mathrm{R} \rightarrow \mathrm{R}$ be defined by

$$
F(x)=\sum_{q_{n}^{<x}} p_{n} \text { or } F(x)=\sum_{q_{n}^{\leqslant} \leqslant x} p_{n}
$$

Then $F$ is $(P A C)$ on $\mathrm{R}$ and, further, $F^{\prime}=0$ a.e. on $\mathrm{R}$.

The following lemma greatly extends Lemma 5.1 in [23]. It is, in fact, more powerful tinan we shall actually need in the sequel.

LEMMA 2.1. Let $f \supseteq I=[a, b] \rightarrow \mathrm{R}$ be such that

(i) $P V(f, I)<\infty$, that is, $f$ is $(P V B)$ on $I$,

(ii) if $f$ is $V B$ on every closed subinterval of an open interval $(x, y) \subseteq I$ and if $f(x+)$ and $f(y-)$ exist (finitely or infinitely), then $f(x) \leqslant f(x+)$ and $f(y-) \leqslant f(y)$, and

(iii) there is a subset $B \subseteq I$ with $|B|=0$ such that, if $x \in I^{0} B$ and $f$ is continuous at $x$, then $D^{+} f(x) \geqslant 0$.

Then, for every set $A \supseteq B$, the function $g(x)=f(x)+P V(f, A \cap[a, x])$ is nondecreasing on $I$. 
Proof. We first assert that, if $f$ is $V B$ on an open interval $J \subseteq I$, then $g$ is nondecreasing on $J$.

Suppose, for a contradiction, that $g(d)<g(c)$ for some $[c, d] \subseteq J$. Choose $\varepsilon>0$ so small that $g(d)<g(c)-\varepsilon(d-c)$. Then, using (2.8), we have $P V(f, A \cap[c, d])=P V(f, A \cap[a, d])-P V(f, A \cap[a, c])=g(d)-f(d)$ $-g(c)+f(c)<F(c)-F(d)$, where

$$
F(x)=f(x)+\varepsilon x \text { for all } x \in I .
$$

But, since $|B \cap[c, d]|=0$ and $F-f$ is $A C$ on $[c, d]$, by (2.4) and (2.2) we have $|F(B \cap[c, d])| \leqslant P V(F, B \cap[c, d])=P V(f, B \cap[c, d]) \leqslant$ $P V(f, A \cap[c, d])$ because $B \subseteq A$. Hence it follows that $0 \leqslant|F(B \cap[c, d])|<F(c)-F(d)$. Then, select any $r \in(F(d), F(c))$ with $r \notin F(B \cap[c, d])$, and set

$$
t=\sup \{x \in(c, d) \mid F(x)>r\} .
$$

Now, by (ii), for all $x \in J$ we have $F(x-)=f(x-)+\varepsilon x \leqslant f(x)+\varepsilon x$ $=F(x)$ and $F(x+)=f(x+)+\varepsilon x \geqslant f(x)+\varepsilon x=F(x)$. In particular, $F(c+) \geqslant F(c)>r$ and $F(d-) \leqslant F(d)<r$, so that $t$ is well defined and $c<t<d$. Then recalling the definition of $t$, we have $F(t) \leqslant F(t+) \leqslant r$ and, hence, further $r \leqslant F(t-) \leqslant F(t)$. Therefore $F$ is continuous at $t$, $F(t)=r \notin F(B \cap[c, d])$ but $t \in(c, d)$, and $D^{+} F(t) \leqslant 0$ since $F(x) \leqslant r=F(t)$ for all $x \in(t, d)$. So, $f$ is continuous at $t$, $t \in I^{0} \backslash B$ and $D^{+} f(t)=D^{+} F(t)-\varepsilon<0$. This is contrary to (iii), and our assertion is proved.

Now, let $E$ denote the set of points of $I$ having no neighbourhood in $I$ on which $g$ is nondecreasing. Then $E$ is closed. clearly $g$ is nondecreasing on any component $(p, q)$ of $I^{0} \backslash E$. Since, further, $P V(f, A \cap[a, x])$ is nondecreasing and bounded on $I$, clearly $f$ is $V B$ on every closed subinterval of $(p, q)$ and both $f(p+)$ and $f(q-)$ exist. Therefore, by (ii), $f(p) \leqslant f(p+)$ and $f(q-) \leqslant f(q)$, whence we readily obtain that $g(p) \leqslant g(p+)$ and $g(q-) \leqslant g(q)$. So, $g$ is in fact nondecreasing on $[p, q]$. In particular, therefore, $E$ is perfect, and $g$ is nondecreasing on $I$ if $E=\varnothing$.

Suppose $E \neq \varnothing$. Since $f$ is $(P V B)$ on $I$, by (2.5) it is (VBG) on $I$. So, by the Baire category theorem $([16], p .54)$, there is an open interval $J \subseteq I$ intersecting $E$ such that $f$ is $V B$ on $E \cap J$. 
Clearly then $g$ is $V B$ on $E \cap J$. Since, further, by above $g$ is nondecreasing on the closure of each component of $J \backslash E$, it follows that $g$ is $V B$ on $J$. Therefore $f$ is $V B$ on $J$. So, by the initial assertion, $g$ is nondecreasing on $J$. This contradicts the condition $E \cap J \neq \varnothing$, and the proof is complete.

REMARK. The lemma remains valid if in (iii) we replace $D^{+} f(x)$ by $D^{-} f(x)$. The initial assertion in the proof can then be verified by setting $t=\inf \{x \in(c, d) \mid F(x)<r\}$.

\section{Abstract limit process}

DEFINITION 3.1. Let $R(+)$ denote the set of ordered pairs $(f, x)$ such that $x \in \mathrm{R}$ and $f \supseteq(x, y) \rightarrow \mathrm{R}$ for some $y>x$. A right-hand limit process in $R$ is a real valued operator $T$ with dom $T \subseteq R(+)$ such that

(i) if $f \supseteq(x, y) \rightarrow \mathrm{R}$ is $(V B G)$ on $(x, y)$ and if the ordinary limit $f(x+)$ exists finitely, then $(f, x) \in \operatorname{dom} T$,

(ii) if $(f, x) \in \operatorname{dom} T$ and if $(g, x) \in R(+)$ is such that $g(t)=f(t)$ for all $t \in(x, y)$ for some $y>x$, then $(g, x) \in$ dom $T$ and, further, $T(g, x)=T(f, x)$,

(iii) if $(f, x),(g, x) \in$ dom $T$ and $p, q \in R$, then $(p f+q g, x) \in$ dom $T$ and, further, $T(p f+q g, x)=p \cdot T(f, x)+q \cdot T(g, x)$, and

(iv) $\lim$ inf $f(t) \leqslant T(f, x) \leqslant \lim \sup f(t)$ for all $(f, x) \in \operatorname{dom} T$. $t \rightarrow x+\quad t \rightarrow x+$

A left-hand limit process in $R$ is defined similarly. By a limit process $T$ in $\mathrm{R}$ we shall mean a pair of a right-hand limit process $T^{+}$ and a left-hand limit process $T^{-}$in $R$, and we shall write $T f(x+)$ for $T^{+}(f, x)$ and $T f\left(x^{-}\right)$for $T^{-}(f, x)$. If $T f(x+), T f(x-)$ and $f(x)$ all exist and are equal to one another, we say that $f$ is $T$-continuous at $x$.

Conventionally, speaking about $T$-continuity of $f$ on a closed interval $[a, b]$, we shall always exempt $T f(a-)$ and $T f(b+)$.

DEFINITION 3.2. A limit process $T$ in $R$ is said to be Darboux, if every function which is $T$-continuous on a closed interval is Darboux (has the intermediate value property) on the interval.

Every orderly connected topology on $R$ induces a Darboux limit process ([23], section 4); in particular, the ordinary, the approximate 
and the proximal limit processes are Darboux. The notions of $C_{n}$-continuity [8], $M_{n}$-continuity [10] and PC-continuity [14] are defined directly in terms of certain types of limits. The reader can easily frame the immediate formal definitions of the corresponding limit processes, in agreement with the above definition. Sargent [19] has shown that the $C_{n}$ - and $M_{n}$-limit processes are Darboux. Again, by definition, a PC-continuous function on an interval is necessarily a finite proximal derivative. Hence by a result of sinharoy ([25], Theorem 4, p. 322), the $P C$-limit process is also Darboux.

Limit processes can also be defined in a natural way in terms of 'selections' [15] and 'bilateral systems of paths' [2], and, more generally, in terms of any topology on $R$ which is such that every point of every open set is an ordinary bilateral limit point of the set. A selective limit process is necessarily Darboux ([15], Theorem B, p. 85), but the others need not be. However, we have the following useful result, which extends Theorem 5.3 in ([2], p. 111).

LEMMA 3.1. Let $T$ be any limit process in $\mathrm{R}$. If $f:[a, b] \rightarrow \mathrm{R}$ is Baire 1 and $T$-continuous on $[a, b]$, then $f$ is Darboux on $[a, b]$.

Proof. By T-continuity, $f$ lies between its ordinary upper and lower unilateral limits on either side in $[a, b]$, and this is precisely the condition for a Baire 1 function to be Darboux [24] (see, also, [1], Theorem 6.1, p. 103).

\section{The (TP)-integral}

Throughout this section, we shall deal with an arbitrary but fixed limit process $T$ in $R$, and with arbitrary functions $f, g \sim I=[a, b] \rightarrow R$.

DEFINITION 4.1. A function $u \supseteq I \rightarrow \mathrm{R}$ is called a (TP)-upper function of $f$ on $I$, in symbols $u \in U(f ; I)$, if

(i) $u(a)=0$,

(ii) $T u(x+)$ exists and $u(x) \leqslant T u(x+)$ for all $x \in[a, b)$,

(iii) $T u(x-)$ exists and $T u(x-) \leqslant u(x)$ for all $x \in(a, b]$,

(iv) $u$ is $(P V B)$ on $I$, and

(v) $(\infty p) u^{\prime} \geqslant f$ a.e. on $I$.

(By (2.5), (oq) $u^{\prime}$ exists finitely a.e. on $I$.) 
A function $\ell \supseteq I \rightarrow R$ is called a (TP)-lower function of $f$ on $I$, in symbols $\ell \in L(f ; I)$, if $-\ell \in U(-f ; I)$.

DEFINITION 4.2. For every $\varepsilon>0$ we define

$$
\begin{aligned}
& U_{\varepsilon}(f ; I)=\{u \in U(f ; I) \mid P V(u, I)<\varepsilon\}, \\
& L_{\varepsilon}(f ; I)=\{l \in L(f ; I) \mid P V(l, I)<\varepsilon\}, \\
& \bar{f}(I ; \varepsilon)=\inf \left\{u(b) \mid u \in U_{\varepsilon}(f ; I)\right\}, \\
& f(I ; \varepsilon)=\sup \left\{l(b) \mid \ell \in L_{\varepsilon}(f ; I)\right\} .
\end{aligned}
$$

If the two monotone limits

$$
\bar{f}(I)=\lim _{\varepsilon \rightarrow 0+} \uparrow \bar{f}(I ; \varepsilon) \text { and } f(I)=\lim _{\varepsilon \rightarrow 0+}+f(I ; \varepsilon)
$$

have a common finite value, then the function $f$ is said to be (TP)integrable on $I$, in symbol $f \in(T P)(I)$, and the common finite limit, denoted $f * I$, is called the definite (TP)-integral of $f$ on $I$.

REMARK. By definition, inf $\varnothing=\infty$ and sup $\varnothing=-\infty$. Therefore, if $U_{\varepsilon}(f ; I)=\varnothing$ for some $\varepsilon>0$ then $\overline{f(I)}=\infty$, and if $L_{\varepsilon}(f ; I)=\varnothing$ for some $\varepsilon>0$ then $f(I)=-\infty$.

THEOREM 4.1. Let $u \in U(f ; I)$ and $\ell \in L(f ; I)$. Then there is a subset $B \subseteq I$ with $|B|=0$ such that, for every $A \supseteq B$, $u(x)-\ell(x)+P V(u-l, A \cap[a, x])$ is nondecreasing and nonnegative on $I$.

Proof. By (2.3), $u-l$ is $(P V B)$ on $I$. Also, for all $x \in[a, b)$ we have $u(x)-\ell(x) \leqslant T u(x+)+T(-l)(x+)=T(u-\ell)(x+)$, and for all $y \in(a, b]$ we have $T(u-l)(y-)=T u(y-)+T(-\ell)(y-) \leqslant u(y)-\ell(y)$. Finally, we have $(o p) u^{\prime} \geqslant f$ a.e. on $I$ and $(o p)(-l)^{\prime} \geqslant-f$ a.e. on $I$. So, there is a subset $B \subseteq I$ with $|B|=0$ such that $(o p)(u-\ell)^{\prime}(x) \geqslant 0$ for all $x \in I^{0} \backslash B$. Hence Lemma 2.1 certainly applies to $u-\ell$, and the required result follows.

COROLLARY 4.1.1. Let $u \in U(f ; I)$ and $\ell \in L(f ; I)$. Then

(i) $u-\ell$ is $V B$ on $I$,

(ii) $u(b)-\ell(b)+P V(u-l, I) \geqslant 0$, and

(iii) $u(b)+P V(u, I) \geqslant \ell(b)-P V(\ell, I)$.

Proof. By Theorem 4.1 with $A=I$, the function $H(x)=u(x)-\ell(x)$ $+P V(u-\ell,[a, x])$ is nondecreasing on $I$. Since, further, $P V(u-\ell,[a, x])$ 
is nondecreasing on $I$, it follows at once that $u-\ell$ is $V B$ on $I$. Also, we have $u(b)-\ell(b)+P V(u-\ell, I)=H(b) \geqslant H(a)=0$; whence again $u(b)+P V(u, I) \geqslant \ell(b)-P V(\ell, I)$, since $P V(u-\ell, I) \leqslant P V(u, I)+P V(\ell, I)$ by (2.1).

From Corollary 4.1.1(iii) we readily obtain:

COROLLARY 4.1.2. We have

(i) $\bar{f}(I) \geqslant \ell(b)-P V(\ell, I)$ for alz $\ell \in L(f ; I)$,

(ii) $f(I) \leqslant u(b)+P V(u, I)$ for all $u \in U(f ; I)$, and

(iii) $\bar{f}(I) \geqslant f(I)$.

Using Theorem 4.1 and its corollaries and, of course, the properties of $T$ and $P V$, the reader can now easily prove:

THEOREM 4.2 .

(i) $f \in(T P)(I)$ if and only if there exist, for every $\varepsilon>0$, at least one $u \in U_{\varepsilon}(f ; I)$ and at least one $\ell \in L_{\varepsilon}(f ; I)$ such that $|u(b)-\ell(b)|<\varepsilon$.

(ii) If $f \in(T P)(I)$ then $\ell(b)-P V(\ell, I) \leqslant f_{* I} \leqslant u(b)+P V(u, I)$ for alz $\ell \in L(f ; I)$ and all $u \in U(f ; I)$.

(iii) If $f \in(T P)(I)$ and if $g=f$ a.e. on $I$, then $g \in(T P)(I)$ and, further, $g * I=f * I$.

(iv) If $f, g \in(T P)(I)$, then $(p f+q g) \in(T P)(I)$ for all $p, q \in \mathrm{R}$ and, further, $(p f+q g) * I=p \cdot(f * I)+q \cdot(g * I)$.

(v) If $J=[a, c]$ and $K=[c, b]$ where $c \in I^{0}$, then $f \in(T P)(I)$ if and only if $f \in(T P)(J) \cap(T P)(K)$, and then $f * I=f * J+f * K$.

We see that when $f \in(T P)(I)$ then $f \in(T P)([a, x])$ for all $x \in I^{0}$. We shall write $F=(T P)(f: I)$ to mean that $f \in(T P)(I)$ and that $F$ is the indefinite (TP)-integral of $f$ on $I$, defined on the entire real line by $F(x)=f *[a, x]$ for $a<x \leqslant b, F(x)=0$ for $x \leqslant a$ and $F(x)=F(b)$ for $x>b$.

THEOREM 4.3. Let $F=(T P)(f: I)$. Then

(i) $u(x)-F(x)+P V(u,[a, x])$ is nondecreasing on $I$ and $V(u-F, I) \leqslant u(b)-F(b)+2 P V(u, I)$ for all $u \in U(f ; I)$, and

(ii) $F(x)-\ell(x)+P V(\ell,[a, x])$ is nondecreasing on $I$ and $V(F-\ell, I) \leqslant F(b)-\ell(b)+2 P V(l, I)$ for all $\ell \in L(f ; I)$.

(In particular, both $u-F$ and $F-\ell$ are $V B$ on $I$.) 
Proof. Similar to the proof of Theorem 5.3 in [23].

The following theorem provides an interesting descriptive definition of the (TP)-integral, similar to those of the Denjoy integrals ([16], p. 241).

THEOREM 4.4. Let $F: \mathrm{R} \rightarrow \mathrm{R}$ be such that $F(x)=0$ for $x \leqslant a$ and $F(x)=F(b)$ for $x>b$. Then $F=(T P)(f: I)$ if and only if $F$ is $T$-continuous and $(P A C)$ on $I$ and $(o p) F^{\prime}=f$ a.e. on $I$ (that is, $F \in U_{\varepsilon}(f ; I) \cap L_{\varepsilon}(f ; I)$ for every $\left.\varepsilon>0\right)$.

Proof. The 'if' part is obvious. To prove the 'only if' part, suppose $F=(T P)(f: I)$.

Consider any $c \in[a, b)$. Given $\varepsilon>0$, we can find $u \in U_{\varepsilon}(f ; I)$ and $\ell \in L_{\varepsilon}(f ; I)$ such that $u(b)-F(b)<\varepsilon$ and $F(b)-\ell(b)<\varepsilon$. Then by Theorem 4.3, for all $x \in I$ we have $-\varepsilon<u(x)-F(x)<2 \varepsilon$ and $-\varepsilon<F(x)-\ell(x)<2 \varepsilon$; also, $u-F$ and $F-\ell$ being $V B$ on $I$ possess finite ordinary right-hand limits at $c$. Since, further, by definition $T u(c+)$ and $T \ell(c+)$ exist and satisfy $u(c) \leqslant T u(c+)$ and $T \ell(c t) \leqslant \ell(c)$, it follows from the definition of $T$ that $T F(c+)$ exists and, further, that

$$
\begin{gathered}
F(c)-3 \varepsilon<u(c)-2 \varepsilon \leqslant T u(c+)-2 \varepsilon \\
=T(u-F)(c+)+T F(c+)-2 \varepsilon \leqslant T F(c+) \leqslant T F(c+)-T(F-\ell)(c+)+2 \varepsilon \\
=T \ell(c+)+2 \varepsilon \leqslant \ell(c)+2 \varepsilon<F(c)+3 \varepsilon .
\end{gathered}
$$

Thus $|T F(c+)-F(a)|<3 \varepsilon$. Since $\varepsilon>0$ is arbitxary, we have $T F(c+)=F(c)$. Similarly, $T F(c-)=F(c)$ for $c \in(a, b]$. Hence $F$ is $T$-continuous on $I$.

The remaining parts of the proof are exactly similar to those for Theorem 5.4 in [23].

COROLLARY 4.4.1. If $F=(T P)(f: I)$, then $F$ is Darboux on $I$.

Proof. Since $F$ is $(P A C)$ on $I$, by $(2.5)$ it is $(V B G)$ on $I$. so $F$ is Baire 1 on $I$. Since, further, $F$ is $T$-continuous on $I$, by Lemma 3.1 it is Darboux on $I$.

Referring to (2.6) and to Corollaries 5.4.1, 5.4.2 in [23] and to the 
remarks preceding them, we omit the simple proofs of the following corollaries.

COROLLARY 4.4.2.

(i) If $f \in(T P)([a, x])$ for $a l l \quad x \in I^{0}$ and if $T E(b-)$ exists, where $F(x)=f *[a, x]$, then $f \in(T P)(I)$ and $f * I=T F(b-)$.

(ii) If $f \in(T P)([x, b])$ for $a l l \quad x \in I^{0}$ and if $T G(a+)$ exists, where $G(x)=f *[x, b]$, then $f \in(T P)(I)$ and $f * I=T G(a+)$.

(iii) If $f \in(T P)(I)$, then $f \mid I$ is measurable and the (TP)-upper and lower functions of $f$ on $I$ are $T$-continuous n.e. (that is, except at a countable number of points) on $I$.

COROLLARY 4.4.3. If $f$ is Lebesgue $(L-)$ integrable on $I$, then it is (TP)-integrable on $I$. Conversely, if $f$ is (TP)-integrable on $I$ and if $g$ is L-integrable on $I$ and either $f \leqslant g$ a.e. on $I$ or $g \leqslant f$ a.e. on $I$, then $f$ is L-integrable on $I$ to the value $f * I$.

THEOREM 4.5 (Integration by parts). Let $F=(T P)(f: I)$ where $F$ is bounded, and let $G=L(g: I)$. Then $f G \in(T P)(I)$ and $(f G) * I=F(b) G(b)$ - $(F g) * I$.

Proof. Since $G$ is the indefinite $L$-integral of $g$ on $I$, it is $A C$ on $I$. Also, by Theorem 4.4, $F$ is (PAC) on $I$. So, by (2.7), $F G$ is $(P A C)$ on $I$. Also, $F G$ is $T$-continuous on $I$. For, given $c \in I, F \cdot(G-G(c))$ is $(V B G)$ on $I$ by $(2.5)$, and it has an ordinary limit 0 at $c$, since $F$ is bounded and $G$ is continuous at $c$. Since, further, $F$ is $T$-continuous at $c$ by Theorem 4.4, it follows from the definition of $T$ that $F G=F \cdot(G-G(c))+F \cdot G(c)$ is $T$-continuous at $c$.

Therefore, by Theorem 4.4 we have $F G=(T P)\left((O Q)(F G)^{\prime}: I\right)$. But $F g$ is $L$-integrable on $I$, since $g$ is and $F$ is bounded and measurable by (2.5). Hence, recalling corollary 4.4 .3 , the required results follow at once by noting that $(c o p)(F G)^{\prime}=f G+F g$ a.e. on $I$.

THEOREM 4.6 (First mean value theorem). Let $H=(T P)(f g: I)$, $G=(T P)(g: I), G(b) \neq 0$, cond either ess-inf $g \geqslant 0$ or ess-sup $g \leqslant 0$. Set $p=H(b) / G(b)$ and $E=\{x \in I \mid g(x) \neq 0\}$. Then either (i) ess-inf $f<p<\operatorname{ess-sup}_{I} f$ or (ii) $f=p$ a.e. on $E,|E|>0$, and $p$ has one of the values ess-inf $f$ or $\operatorname{ess-sup}_{I} f . \quad(G(b)=0$ implies $H(b)=0$. ) 
Proof. Similar to the proof of Theorem 5.6 in [23].

THEOREM 4.7 (Second mean value theorem). Let $F=(T P)(f: I)$ where $F$ is bounded, and let $G=(T P)(g: I), G(b) \neq 0$, and either ess-inf $g \geqslant 0$ or ess-sup ${ }_{I} g \leqslant 0$. Then $f G \in \operatorname{TP}(I)$ and there is a point $c \in I$ such that $(f G) * I=G(b)(F(b)-F(c))$ and such that either (i) $\inf _{I} F<F(c)<\sup _{I} F$ or (ii) $F=F(c)$ a.e. on $E=\{x \in I \mid g(x) \neq 0\}$, $|E|>0$, and $F(c)$ has one of the values $\inf _{I} F$ or $\sup _{I} F$.

Proof. Similar to the proof of Theorem 5.7 in [23].

THEOREM 4.8. Let $\left\{I_{n}=\left[a_{n}, b_{n}\right]\right\}$ be a sequence of nonoverlapping closed subintervals of $I$ and $G=U_{n} I_{n}^{0}$. Suppose $F_{n}=(T P)\left(f: I_{n}\right)$ cand $\sum_{n}\left|F_{n}\left(b_{n}\right)\right|<\infty$. Defining $S(x)=\sum_{n} F_{n}(x)$, suppose further that both $T S(x-)$ and TS $(x+)$ exist for all $x$ and that

$$
\sum_{r \in \mathrm{R}}\{|O(S, r-)|+|O(S, r+)|\}<\infty,
$$

where

$$
O\left(S, r^{-}\right)=T S(r-)-S(r), \quad O(S, r+)=S(r)-T S(r+) .
$$

Then we have $F=(T P)\left(f_{G}: I\right)$ where $F$ is defined on $\mathrm{R}$ by

$$
F(x)=S(x)+\sum_{r<x} O(S, r-)+\sum_{r<x} O(S, r+) .
$$

Proof. The condition $\sum\left|F_{n}\left(b_{n}\right)\right|<\infty$ implies that $\sum F_{n}(x)$ is absolutely convergent for all $x$, and so $S$ is well defined. Also, the condition $\left({ }^{*}\right)$ implies that $S$ is $T$-continuous n.e. and that $F$ is well defined.

Now, noting that $T S(x-)$ and $T S(x+)$ exist for all $x$ and that the second and third parts in the expression for $F$ are $V B$ on $R$, it follows from the definition of $T$ that both $T F(x-)$ and $T F(x+)$ exist for all $x$ and, further,

$$
\begin{aligned}
T F(x-) & =T S(x-)+\sum_{r<x} O(S, r-)+\sum_{r<x} O(S, r+) \\
& =S(x)+\sum_{n<\infty} O(S, r-)+\sum_{n<r} O(S, r+)=F(x)
\end{aligned}
$$


and

$$
\begin{aligned}
T F(x+) & =T S(x+)+\sum_{r \leqslant x} O(S, r-)+\sum_{r \leqslant x} O(S, r+) \\
& =S(x)+\sum_{r<x} O(S, r-)+\sum_{r<x} O(S, r+)=F(x) .
\end{aligned}
$$

Again, since $F(x)=F\left(a_{n}\right)+F_{n}(x)$ for all $x \in I_{n}$, it follows from Theorem 4.4 that, for each $n, F$ is $(P A C)$ on $I_{n}$ and $(o p) F^{\prime}=(\alpha O) F_{n}^{\prime}$ $=f$ a.e. on $I_{n}$. On the other hand, for all $x \in I \backslash G$ we have

$$
F(x)=\sum_{n} \sum_{n} F_{n}\left(b_{n}\right)+\sum_{r \leqslant x} O(S, r-)+\sum_{r<x} O(S, r+),
$$

which by (2.10) implies that $F$ is $(P A C)$ on $I \backslash G$ and that $(a P) F^{\prime}=0$ a.e. on $I \backslash G$, since $G$ has density 0 a.e. on $I \backslash G$.

Summing up, we see that $F$ is $T$-continuous on $I$, it is $(P A C)$ on $I$ by $(2.6)$, and $(a p) F^{\prime}=f_{G}$ a.e. on $I$; which by Theorem 4.4 completes the proof, since evidently $F(x)=0$ for $x \leqslant a$ and $F(x)=F(b)$ for $x>b$.

We are now ready to prove the much coveted analogue of the Marcinkiewicz theorem for the ordinary Perron integral ([16], (3.13), p. 253; [28], p. 648; [20]). Skvorcov [26] obtained a similar result for the CP-integral of Burkill.

THEOREM 4.9. If there exist at least one $u \in U(f ; I)$ and at least one $\ell \in L(f ; I)$, and if $f \mid I$ is measurable, then $f \in(T P)(I)$.

Proof. Let $E_{0}$ denote the set of points $x \in I$ such that $f \notin(T P)$ $(I \cap J)$ for all closed intervals $J$ with $x \in J^{\theta}$. Clearly $E_{0}$ is closed, and it is enough to derive a contradiction by assuming that $E_{0} \neq \emptyset$.

We first show that, if $[p, q]$ is the closure of a component of $I \backslash E_{0}$, then $f \in(T P)([p, q])$. Evidently $f \in(T P)(J)$ for every closed interval $J \subseteq(p, q)$. Fix $c \in(p, q)$, and set $F(x)=f *[c, x]$ for $x \in(c, q)$ and $F(x)=0$ for $x \leqslant c$. Clearly $u_{1}=u-u(c) \in U(f ;[c, b])$ and $\ell_{1}=\ell-\ell(c) \in L(f ;[c, b])$. Therefore Theorem 4.3 implies that the 
function $h(x)=u_{1}(x)-F(x)+P V\left(u_{1},[c, x]\right)$ is nondecreasing on $(c, q)$. Since, further, by Corollary 4.1.1(i) and Theorem $4.2(i i)$ we have $h(x)=\left\{u_{1}(x)-\ell_{1}(x)\right\}+\left\{\ell_{1}(x)-F(x)\right\}+P V\left(u_{1},[c, x]\right) \leqslant V\left(u_{1}-\ell_{1},[c, b]\right)+$ $P V(\ell,[c, \dot{x}])+P V\left(u_{1},[c, x]\right) \leqslant V(u-\ell, I)+P V(\ell, I)+P V(u, I)<\infty$, it follows that the monotone limit $h\left(q^{-}\right)$is finite. Since, further, $T u_{1}(q-)$ exists and $P V\left(u_{1},[c, x]\right)=P V(u,[c, x])$ is nondecreasing and bounded on $(c, q)$, it follows from the definition of $T$ that $T F(q-)$ exists. Hence $f \in(T P)([c, q])$ by Corollary 4.4.2(i). Similarly $f \in(T P)([p, c])$. Hence $f \in(T P)([p, q])$. In particular, therefore, $E_{0}$ is perfect.

Now, by (2.5) both $u$ and $\ell$ are $(V B G)$ on $I$. So, using Baire theorem we can find an interval $J=[c, d]$ with $c, d \in E_{0}$, such that $E_{0} \cap J^{0} \neq \varnothing$ and such that both $u$ and $\ell$ are $V B$ on $E=E_{0} \cap J$. Let $\left\{J_{n}=\left[p_{n}, q_{n}\right\}\right\}$ denote the sequence of closures of the components of $J \backslash E$, and let $u_{0}$ and $\ell_{0}$ denote respectively the functions obtained by extending $u \mid E$ and $\ell \mid E$ linearly on each of the intervals $J_{n}$. Then both $u_{0}$ and $\ell_{0}$ are $V B$ on $J$. Evidently $(a p) u^{\prime}=u_{0}^{\prime}$ and $(a p) \ell^{\prime}=\ell_{0}^{\prime}$, a.e. on $E$. since, further, $\left.(o p) u^{\prime} \geqslant f \geqslant(o q)\right) \ell^{\prime}$ a.e. on $E$ and since $f \mid E$ is measurable, it follows that $f_{E}$ is $L$-integrable. In particular $f_{E} \in(T P)(J)$ (and, hence, $E$ is nondense).

We shall now verify the hypotheses of Theorem 4.8 on $J$. As already shown, $f \in(I P)\left(J_{n}\right)$ for each $n$. Writing $E_{n}=(T P)\left(f: J_{n}\right)$, Theorem $4.2\left(\right.$ ii) implies that $\ell\left(q_{n}\right)-\ell\left(p_{n}\right)-P V\left(\ell, J_{n}\right) \leqslant F_{n}\left(q_{n}\right) \leqslant u\left(q_{n}\right)-u\left(p_{n}\right)+$ $P V\left(u, J_{n}\right)$ for all $n$. Therefore $\left[\left|F_{n}\left(q_{n}\right)\right|<\infty\right.$, since both $u$ and $\ell$ are $V B$ on $E$ and $(P V B)$ on $I$. Hence the function $S(x)=\sum_{n}(x)$ is well defined. We further define

$$
H(x)=u(x)-S(x)+P V(u,[c, x]), x \in J .
$$

Now, for all $x \in J_{k}=\left[p_{k}, q_{k}\right]$ we clearly have 


$$
H(x)+\sum_{q_{n}<p_{k}} F_{n}\left(q_{n}\right)-P V\left(u,\left[c, p_{k}\right]\right)=u(x)-F_{k}(x)+P V\left(u,\left[p_{k}, x\right]\right),
$$

which is nondecreasing on $J_{k}$ by Theorem 4.3. Therefore $H$ is nondecreasing on $J_{k}$ for each $k$. Further, since for all $x \in E$ we have

$$
H(x)=u_{0}(x)-S_{0}(x)+P V(u,[c, x]), S_{0}(x)=\sum_{q_{n} \leqslant x} F_{n}\left(q_{n}\right),
$$

clearly $H$ is $V B$ on $E$. Hence $H$ is in fact $V B$ on $J$. Clearly then $u-S$ is $V B$ on $J$. Since, further, $T u(x t)$ and $T u(y-)$ exist for all $x \in[c, d)$ and $y \in(c, d]$, it follows from the definition of $T$ that both $T S(x+)$ and $T S(y-)$ exist.

Now, let $r \in E$ be a limit point of $E$ on the right. Then, since $u-S$ is $V B$ on $J$ and $u-S=u_{0}-S_{0}$ on $E$, we clearly have $T u(r+)-T S(r+)=T(u-S)(r+)=(u-S)(r+)=\left(u_{0}-S_{0}\right)(r+)=u_{0}(r+)-S_{0}(r+)=$ $u_{0}(p+)-S_{0}(r)$. Therefore,

$$
S_{0}(r)-T S(r+)=u_{0}(r+)-T u(r+)
$$

Similarly, showing that $S-\ell$ is $V B$ on $J$, we get

$$
S_{0}(r)-T S(r+)=\ell_{0}(r+)-T \ell(r+) \text {. }
$$

But, since $r \in E$, we have $S_{0}(r)=S(r), T u(r+) \geqslant u(r)=u_{0}(r)$ and $T \ell(r+) \leqslant \ell(r)=\ell_{0}(r)$. It follows therefore that $\ell_{0}(r+)-\ell_{0}(r) \leqslant S(r)$ $-T S(p+) \leqslant u_{0}(x+)-u_{0}(r)$, whence

$$
|O(S, r+)| \leqslant\left|u_{0}(r+)-u_{0}(r)\right|+\left|\ell_{0}(r+)-\ell_{0}(r)\right| .
$$

Again, since $S(x)=S\left(p_{k}\right)+F_{k}(x)$ for $x \in J_{k}$, for all $x \in \mathrm{U}_{n}\left[p_{n}, q_{n}\right)$ we clearly have $T S(x+)=S(x)$. So (1) holds in fact for all $r \in[c, d)$. similarly, for all $r \in(c, d]$ we have

$$
|O(S, r-)| \leqslant\left|u_{0}(r)-u_{0}(r-)\right|+\left|\ell_{0}(r)-\ell_{0}(r-)\right| .
$$

since $u_{0}$ and $\ell_{0}$ are $V B$ on $J$, and since $S(x)=0$ for $x \leqslant c$ 
and $S(x)=S(d)$ for $x>d$, it follows from (1) and (2) that $\sum_{r \in \mathrm{R}}|O(S, r+)|<\infty$ and $\sum_{r \in \mathrm{R}}|O(S, r-)|<\infty$. Hence by Theorem 4.8, $f_{G} \in(T P)(J)$ where $G=\bigcup_{n} J_{n}^{0}$. since, moreover, $f_{E} \in(T P)(J)$ and $f_{J}=f_{E}+f_{G}$, it follows that $f \in(T P)(J)$. This contradicts the condition $E_{0} \cap J^{0} \neq \varnothing$, and the proof is complete.

COROLLARY 4.9.1. Suppose $F: I \rightarrow \mathrm{R}$ is $(P V B)$ on $I$. Defining $F(x)=F(a)$ for $x<a$ and $F(x)=F(b)$ for $x>b$, suppose further that both $T F(x-)$ and $T F(x+)$ exist for all $x \in I$, and that $\sum_{r \in I}|O(F, r-)|<\infty$ and $\sum_{r \in I}|O(F, r+)|<\infty$. Then $(a p) F^{\prime} \in(T P)(I)$.

Proof. By (2.5), (ap) $F^{\prime}$ exists finitely a.e. on $I$ and it is measurable ([9], Lemma 3, p. 349). Then the proof follows by verifying (which, by now, must be easy to the reader) that the functions

$$
u(x) \equiv \ell(x)=F(x)-F(a)+\sum_{r<x} O(F, r-)+\sum_{r<x} O(F, r+)
$$

satisfy the hypotheses of 'Theorem 4.9 with $f=(a p) F^{\prime}$.

COROLLARY 4.9.2. Let $F$ be as in Corolzary 4.9.1. Then $F$ can be represented as $F=G+H$, where $G$ is $T$-continuous and (PAC) on $I$ and $H$ is $(P V B)$ on $I$ and $(o p) H^{\prime}=0$ a.e. on $I$. Also, this representation is unique up to an additive constant.

Proof. By Corollary 4.9.1, (ap $) F^{\prime} \in(T P)(I)^{\circ}$. Then it is enough to take $G=(T P)\left((c Q p) F^{\prime}: I\right)$ and $H=F-G$.

\section{Relation with other integrals}

From (2.9) and Theorem 4.4 it follows that, for the ordinary limit process $T$ our (TP)-integral is equivalent to the Denjoy integral in the wide sense ([16], p. 241). When $T$ is the approximate limit process, it has been shown in $([23], p .352)$ that the (TP)-integral is substantially more general than the various known approximately continuous integrals ([6], [12], [13], [27]).

Again, it is known [10] that the $G M_{n}$-integral includes the $C_{n} P-$ integral, which is equivalent to the $C_{n} D$ - and $V_{n} D$-integrals $([18],[29])$. 
Also, if $F=G M_{n}(f: I)$ then. by definition $F$ is $M_{n}$-continuous and ( $A C G$ ) on $I$ and $(a p) F^{\prime}=f$ a.e. on $I$. Since, by (2.9), (ACG) implies $(P A C)$, it follows that $C_{n} P=C_{n} D=V_{n} D \subseteq(T P)$ when $T=C_{n}$ and that $G M_{n} \subseteq(T P)$ when $T=M_{n}$. The $n$th order Perron integral, $P^{n}$, of Bullen [3] is equivalent to that of Bullen and Mukhopadhyay (15], Theorem 27. p. 56), and it is closely related to the $C_{n-1} P$-integral. In particular $P^{n}$-integrability is equivalent to $C_{n-1} P$-integrability ([3], Theorem 16, p. 228). Hence, $P^{n}$-integrability implies (TP)-integrability with $T=C_{n-1}$. Finally, if $F=(P C D)(f: I)$ then by definition ([14], p. 102) $F$ is $P C$-continuous and $(A C G)$ on $I$ and $(a p) F^{\prime}=f$ a.e. on $I$. Therefore $(P C D) \subseteq(T P)$ when $T=P C$.

\section{References}

[1] A.M. Bruckner and J.G. Ceder, "Darboux continuity", Jahresber. Deutsch. Math.-Verein. 67 (1965), 93-117.

[2] A.M. Bruckner, R.J. O'Malley and B.S. Thomson, "Path derivatives: A unified view of certain generalized derivatives", Trans. Amer. Math. Soc. 283 (1984), 97-125.

[3] P.S. Bullen, "The $P^{n}$-integral", J. Austral. Math. Soc. 14 (1972), 219-236.

[4] P.S. Bullen and C.M. Lee, "On the integrals of Perron type", Trans. Amer. Math. Soc. 182 (1973), 481-501.

[5] P.S. Bullen and S.N. Mukhopadhyay, "Peano derivatives and general integrals", Pacific J. Math. 47 (1973), 43-58.

[6] J.C. Burkill, "The approximately continuous Perron integral", Math. Zeit. 34 (1932), 270-278.

[7] J.C. Burkill, "The Cesàro-Perron integral", Proc. London Math. Soc., (2) 34 (1932), 314-322.

[8] J.C. Burkill, "The Cesàro-Perron scale of integration", Proc. London Math. Soc., (2) 39 (1935), 541-552.

[9] J.C. Burkill and U.S. Haslam-Jones, "The derivates and approximate derivates of measurable functions", Proc. London Math. Soc., (2) 
$32(1931), 346-355$.

[10] H.W. Ellis, "Mean-continuous integrals", Canad. J. Math. 1 (1949), $113-124$.

[11] H.W. Ellis, "On the compatibility of the approximate Perron and Cesàro-Perron integrals", Proc. Amer. Math. Soc. 2 (1951), 396-397.

[12] Y. Kubota, "An integral of the Denjoy type", Proc. Japan Acad. 40 (1964), 713-717.

[13] Y. Kubota, "An integral of the Denjoy type. II", Proc. Japan Acad. $42(1966), 737-742$.

[14] R.K. Nath and M.K. Bose, "On the proximal Cesàro-Denjoy integral", Soochow J. Math. 10 (1984), 99-110.

[15] R.J. O'Malley, "Selective derivates", Acta Math. Acad. Sci. Hungar. 29 (1977), 77-97.

[16] s. Saks, Theory of the integral (2nd rev. ed., PWN, Warsaw, 1937).

[17] W.L.C. Sargent, "A descriptive definition of Cesàro-Perron integrals", Proc. London Math. Soc., (2) 47 (1941), 212-247.

[18] W.L.C. Sargent, "On generalized derivatives and Cesàro-Denjoy integrals", Proc. London Math. Soc., (2) 52 (1951), 365-376.

[19] w.L.C. Sargent, "Some properties of $C_{\lambda}$-continuous functions", $J$. London Math. Soc. 26 (1951), 116-121.

[20] D.N. Sarkhel, "A criterion for Perron integrability", Proc. Amer. Math. Soc. 71 (1978), 109-112.

[21] D.N. Sarkhel, "Topological aspect of Cesàro-continuity", Proc. Indian Sc. Congress, Abstract 14 (1981).

[22] D.N. Sarkhel and A.K. De, "The proximally continuous integrals", $J$. Austral. Math. Soc. Ser. A. 31 (1981), 26-45.

[23] D.N. Sarkhel and A.B. Kar, "(PVB) functions and integration", $J$. Austral. Math. Soc. Ser. A. 36 (1984), 335-353.

[24] H.K. Sen, "Darboux's property and its applications", Proc. Benares Math. Soc. (N.S.) 2 (1940), 17-23. 
[25] M. Sinharoy, "Remarks on Darboux and mean value properties of approximate derivatives", Comment. Math. Prace Mat. 23 (1983), 315-324.

[26] V.A. Skvorcov, "Nekotorye svoístva CP-integrala", Mat. Sb. 60 (1963), 304-324; English transl., Amer. Math. Soc. Translations, (2) 54 (1966), 231-254.

[27] G. Sunouchi and M. Utagawa, "The generalized Perron integrals", Tôhoku Math. J. 1 (1949), 95-99.

[28] G. Tolstoff, "Sur l'intégrale de Perron", Mat. Sb. 5 (1939), 647-659.

[29] S. Verblunsky, "On a descriptive definition of Cesàro-Perron integrals", J. London Math. Soc., (2) 3 (1971), 326-333.

Department of Mathematics,

University of Kalyani,

Kalyani, west Bengal,

India 741235. 